\title{
EDITORIAL
}

(O) PAEDIATRIC CANCER

\section{The little C}

A cancer diagnosis is life changing at any age, but a diagnosis at a young age can alter an individual's life path completely. A child thrown into the world of hospitals, tests and treatments also opens technical challenges from the medical perspective-dosing issues, preservation of growing structures, surgery on tiny bodies-as well as psychosocial challenges. At the time of primary treatment, the whole family is affected, from the parents of the sick child to the siblings left at home. For cancer survivors, the long-term and/or late adverse effects of chemotherapy and radiotherapy include impaired bone growth, cognitive and learning deficits, impaired fertility and an increased risk of secondary malignancies later in life. It's easy to see how life as one knows it can be upended.

Perhaps the saving grace of paediatric cancer is its rarity: an estimated 175,000 children $<15$ years in age were diagnosed in 2008 globally. However, the incidence of childhood cancers-which include leukaemias, lymphomas, brain and spinal tumours, sarcomas and kidney tumours-is rising. Despite this increase, in high-income countries, childhood cancers are associated with a very high level of treatment success (approaching 90\%), with many childhood-cancer survivors reaching adulthood. By contrast, overall-survival rates are much lower in regions with low or middle incomes: 5-year survival rates are as low as 40-60\% in Egypt, Honduras and Venezuela; and a paltry $5-10 \%$ in Bangladesh, the Philippines, Senegal, Tanzania and Vietnam. This disparity must be and is being addressed: paediatric oncology today is focusing on better treatments without long-term adverse effects, modified regimens for low-resource settings, personalized treatments, programmes to help patients adhere to their prescribed regimens and support for families.

Indeed, paediatric oncology is a bustling field with a wide remit that includes genomic studies, development of cell-based treatments, surgical innovations and palliative care. This focus issue was commissioned to look

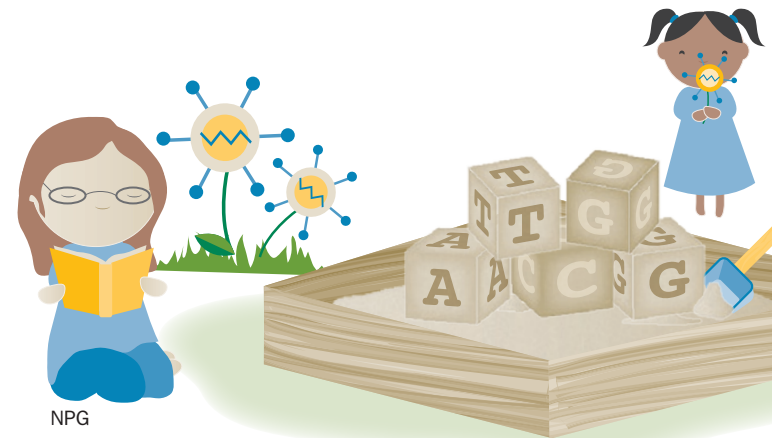

at a number of these key issues and the cutting-edge research surrounding the treatment of arguably our most vulnerable patients.

Crystal Mackall et al. discuss efforts that are underway to generate and test new immunotherapies, such as adoptive cell transfer, in a wide array of paediatric cancers. The challenges in this field include expanding the availability of technically demanding platforms to optimize the management of treatment-related toxicities. Next, Garrett Brodeur and Rochelle Bagatell examine whether a better understanding of the mechanisms of spontaneous regression of neuroblastoma might help to identify the optimal therapeutic approaches for related tumours that progress in spite of intensive multimodal treatment. Amar Gajjar and Giles Robinson take a closer look at medulloblastoma, a brain cancer that mainly arises during infancy and childhood that has seen a flurry of basic and translational research in recent years. Now considered a group of diseases comprising at least four distinct tumour subtypes, each medulloblastoma has characteristic molecular signatures and clinical features that exert influence on several aspects of the disease, including treatment and survival.

David Poplack and colleagues describe the Passport for Care, a tool that was developed to facilitate clinical decision-making and guide follow-up care individualized to the childhood-cancer survivor. On the social side of medicine, David Malkin and colleagues share their views on how the emergence of new DNA-sequencing technologies and cancer-surveillance strategies is altering the framework for the clinical management of hereditary cancer. They discuss genetic counselling and disclosure issues, and propose strategies for approaching ethical dilemmas. Finally, Peter C. Adamson, Peter Houghton, Giorgio Perilongo and Kathy Pritchard-Jones took the time to answer our questions about drug development in paediatric oncology. Although 120 cancer drugs gained FDA approval in the years between 1948 and 2003, only 30 have been used in children. This slow rate of development must be addressed in a meaningful way if we are to make progress in the most-pressing settings in childhood cancer.

All of the Focus articles can be accessed for free until the end of May 2015 at www.nature.com/nrclinonc/ focus/paediatric-cancer. Produced with support of a grant from Bristol-Myers Squibb. Nature Publishing Group carries sole responsibility for the editorial content.

doi:10.1038/nrclinonc.2014.176
44 ...a

diagnosis

at a young age can alter one's life path completely 77
Mina Razzak is the former Senior Editor of Nature Reviews Clinical Oncology and Chief Editor of Nature Reviews Disease Primers.

Competing interests The author declares no competing interests. 